\title{
Tenofovir Cons the Kidneys: A Case of Acquired Fanconi
}

\author{
Ritu Nahar, MD and Emma Lundsmith, MD
}

\section{INTRODUCTION}

Proximal (Type 2) renal tubular acidosis (RTA) is a relatively rare diagnosis, especially in adults. It is characterized by a reduction in proximal bicarbonate reabsorption resulting in urinary bicarbonate wasting. Proximal RTA can also be associated with additional defects in proximal tubular function including impaired reabsorption of phosphate, glucose, uric acid, and amino acids. Generalized proximal tubular dysfunction is termed Fanconi syndrome. While there are primary causes of Fanconi syndrome including sporadic and familial sources, this syndrome can also be acquired. Two major culprits include monoclonal gammopathies resulting in increased excretion of immunoglobulin light chains and drug-induced nephrotoxicity to the proximal tubules. ${ }^{1}$ Tenofovir disoproxil fumarate (TDF) is one such established nephrotoxic agent associated with Fanconi syndrome, likely because it is excreted through the kidney via active tubular secretion. ${ }^{2-4}$ This case demonstrates a classic presentation of tenofovir-induced Fanconi syndrome complicated by respiratory repercussions of hypophosphatemia, and also describes tenofovir alafenamide (TAF), a novel formulation with reduced renal toxicity.

\section{CASE PRESENTATION}

A 63-year-old male and active smoker with human immunodeficiency virus (HIV) on Atripla (efavirenz/ emtricitabine/TDF), intermittent alcohol abuse for years, severe chronic obstructive pulmonary disease (COPD) on no home oxygen presented with three months of progressive dyspnea on exertion with ambulatory dysfunction. The patient endorsed dyspnea after ambulating 5-10 feet, with associated diffuse body weakness. He noted frequent falls requiring use of a walker. Over the six weeks prior to presentation, he had been treated multiple times for presumed COPD exacerbations with attempts to optimize his triple inhaler therapy. He noted minimal improvement in his respiratory symptoms despite these interventions. Three months prior to presentation, he had been functionally independent.

He was diagnosed with HIV 20 years prior, and had achieved virologic suppression on Atripla without any side effects. His history was also significant for coronary artery disease with non-ST-elevation myocardial infarction in
2012, prior stroke of the corona radiata in 2012 . hypertension, and Barrett's esophagus. He endorsed increased alcohol consumption in the last few months to three drinks daily. He was an active smoker using about half a pack of cigarettes per day. He denied recreational drug use.

He reported no history of fever, chills, diarrhea, recent travel, cough, sputum production, chest pain, lower extremity swelling, orthopnea, or paroxysmal nocturnal dyspnea.

On admission, the patient had a blood pressure of 110/70 $\mathrm{mmHg}$ with negative orthostatics, oxygen saturation of $96 \%$ on room air at rest but $79 \%$ with ambulation. He subsequently developed hypoxia at rest as well. On exam, the patient had diffusely decreased breath sounds with no appreciable wheezes, crackles, or rales. He had a regular S1 and S2 with no jugular venous distension or peripheral edema. Neurological exam was grossly unremarkable with 5/5 strength in all extremities and intact sensation.

Labs were significant for serum bicarbonate $16 \mathrm{meq} / \mathrm{L}$, lactate $2.5 \mathrm{mmol} / \mathrm{L}$, magnesium $0.8 \mathrm{mg} / \mathrm{dL}$, phosphate $0.8 \mathrm{mg} / \mathrm{dL}$, albumin $3.6 \mathrm{mg} / \mathrm{dL}$, hemoglobin $8.3 \mathrm{~g} / \mathrm{dL}$, CD4 173 with undetectable viral load. Urinalysis revealed $3+$ glucose and $\mathrm{pH}$ 7.0. Urine phosphate $27 \mathrm{mg} / \mathrm{dL}$ and creatinine $43 \mathrm{mg} / \mathrm{dL}$.

Transthoracic echocardiogram demonstrated an ejection fraction of $70 \%$ with normal left ventricular size and function. Chest $x$-ray had no focal consolidation or edema. Computed tomography (CT) angiography of the chest was negative for acute pulmonary embolism. Non-contrast CT head demonstrated no intracranial hemorrhage, masses, or lesions.

\section{DIFFERENTIAL DIAGNOSIS}

This patient's constellation of symptoms including progressive shortness of breath, ambulatory dysfunction, normal anion gap metabolic acidosis with hypophosphatemia and hypomagnesemia was initially challenging to unify under one diagnosis.

His primary complaint of progressive shortness of breath had a broad differential. Given his COPD history and new oxygen requirement, progression of his underlying 
disease was considered. The timeline of his symptoms and objective physical exam did not support an acute exacerbation. CT angiogram ruled out pulmonary embolism. Given his history of coronary artery disease, heart failure was also considered. However, this was not corroborated by the patient's physical exam and the echocardiogram demonstrated normal ejection fraction. Thus, we hypothesized that the patient's progressive shortness of breath was secondary to hypophosphatemia-induced respiratory muscle fatigue in the setting of severe COPD.

The differential for the patient's ambulatory dysfunction included orthostatic hypotension, ethanol abuse, and a new mass lesion or stroke given his prior cerebrovascular history. His neurological exam was unremarkable, orthostatics were normal, and CT head was unrevealingmaking his falls likely secondary to weakness as opposed to an intracranial insult. Thus, there may have been a component of hypophosphatemia-induced muscle dysfunction.

Normal anion gap metabolic acidosis can be caused by renal or gastrointestinal loss of bicarbonate, or with decreased renal acid excretion. Loss of bicarbonate is seen with Type 2 RTAs, diarrhea, carbonic anhydrase inhibitors, and toluene inhalation (glue sniffing). ${ }^{5}$

The patient's severe hypophosphatemia and hypomagnesemia was initially attributed to malnutrition in the setting of alcohol abuse. However persistent deficiencies despite intravenous repletion compounded with the normal anion gap metabolic acidosis suggested generalized proximal renal tubular dysfunction.

\section{OUTCOME AND FOLLOW-UP}

Fanconi syndrome was eventually suspected and attributed to TDF. This suspicion was further supported by a urine $\mathrm{pH}$ of $7,3+$ glucosuria, and increased fractional excretion of filtered phosphate. Atripla (which contains TDF) was discontinued and the patient was switched to Descovy (emtricitabine and TAF) and Tivicay (dolutegravir).

The patient's generalized weakness, dyspnea, and hypoxia improved with normalization of his serum bicarbonate, phosphate, and magnesium. He was discharged with oral phosphorus and magnesium supplementation. At his most recent pulmonary follow-up, he had normal ambulatory oximetry with increased functionality.

\section{DISCUSSION}

Tenofovir disoproxil fumarate, the first approved oral prodrug of tenofovir, has been successfully used in combination antiretroviral therapy for HIV treatment since 2001. However, as seen in this case and established in the literature, TDF can cause clinically significant renal toxicity.
This is thought to be secondary to high circulating plasma levels of tenofovir which is then excreted through the kidney via active tubular secretion. Tenofovir alafenamide, a novel oral prodrug of tenofovir, is metabolized to tenofovir intracellularly rather than in plasma. This results in higher intracellular concentrations of the active metabolite compared to TDF. Consequently, the therapeutic dose required for TAF is less than one-tenth of the dose of TDF. ${ }^{6}$ The resulting $90 \%$ reduction in plasma tenofovir concentrations is believed to be responsible for the improved renal safety demonstrated in several phase 2 and 3 randomized-controlled trials. ${ }^{7}$ A recent meta-analysis demonstrated that both TDF-containing regimens and TAF-containing regimens have high and comparable rates of virologic suppression. ${ }^{8}$ While promising, the long-term clinical significance of TAF still needs to be assessed in prospective cohorts.

\section{KEY POINTS}

This case illustrates the renal side effects of TDF-induced Fanconi syndrome, highlighting the manifestations of hypophosphatemia. Recognizing this potential side effect and switching patients to a TAF-containing regimen is important to prevent renal injury.

\section{REFERENCES}

1. Messiaen T, Deret S, Mougenot B, et al. Adult Fanconi syndrome secondary to light chain gammopathy. Clinicopathologic heterogeneity and unusual features in 11 patients. Medicine (Baltimore). 2000;79:135.

2. Izzedine H, Launay-Vacher V, Isnard-Bagnis C, Deray G. Drug-induced Fanconi's syndrome. Am J Kidney Dis. 2003:41:292-309.

3. Monteagudo-Chu MO, Chang MH, Fung HB, Bräu N: Renal toxicity of long-term therapy with tenofovir in HIV-infected patients. J Pharm Pract. 2012;25:552-559

4. Rifkin BS, Perazella MA: Tenofovir-associated nephrotoxicity: Fanconi syndrome and renal failure. Am J Med. 2004:117:282-284.

5. Carlisle EJ, Donnelly SM, Vasuvattakul S, et al. Glue-sniffing and distal renal tubular acidosis: sticking to the facts. J Am Soc Nephrol. 1991;1:1019.

6. Lee WA, He GX, Eisenberg E, et al. Selective intracellular activation of a novel prodrug of the human immunodeficiency virus reverse transcriptase inhibitor tenofovir leads to preferential distribution and accumulation in lymphatic tissue. Antimicrob Agents Chemother. 2005;49:1898-1906

7. Mills A, Arribas JR, Andrade-Villanueva J, et al. Switching from tenofovir disoproxil fumarate to tenofovir alafenamide in antiretroviral regimens for virologically suppressed adults with HIV-1 infection: a randomised, active-controlled, multicentre, open-label, phase 3, non-inferiority study. Lancet Infect Dis. 2016;16:43-52.

8. Wang H, Lu X, Yang X, Xu N. The efficacy and safety of tenofovir alafenamide versus tenofovir disoproxil fumarate in antiretroviral regimens for HIV-1 therapy: Meta-analysis. Medicine (Baltimore). 2016:95(41):e5146. 